\title{
Comparison of Actuator Line Method and Full Rotor Geometry Simulations of the Wake Field of a Tidal Stream Turbine
}

\author{
Xiang-feng Lin ${ }^{1,2}$, Ji-sheng Zhang ${ }^{1,2, *}$, Yu-quan Zhang ${ }^{2,3, * \mathbb{D}}$, Jing Zhang ${ }^{1,2}$ and Sheng Liu 4 \\ 1 Key Laboratory of Coastal Disaster and Defence (Hohai University), Ministry of Education, Nanjing 210024, \\ China; linxiangfeng@hhu.edu.cn (X.-f.L.); 15950527337@163.com (J.Z.) \\ 2 College of Harbor, Coastal and Offshore Engineering, Hohai University, Nanjing 210024, China \\ 3 College of Energy and Electrical Engineering, Hohai University, Nanjing 210024, China \\ 4 College of Civil and Transportation Engineering, Hohai University, Nanjing 210024, China; \\ liusheng3131@163.com \\ * $\quad$ Correspondence: jszhang@hhu.edu.cn (J.-s.Z.); zhangyq@hhu.edu.cn (Y.-q.Z.); Tel.: +86-025-8378-6619 (J.-s.Z.)
}

Received: 14 January 2019; Accepted: 13 March 2019; Published: 18 March 2019

\begin{abstract}
This study aims to investigate the wake characteristics of a horizontal axis tidal stream turbine supported by a monopile using a numerical approach. Computational fluid dynamics (CFD) simulations based on the open source software OpenFOAM have been performed to enhance understanding of a turbine's wake. The numerical simulations adopt both the actuator line method and the full rotor geometry method. The numerical results are found to be consistent with experimental data, although some discrepancies are observed at a distance of one rotor diameter downstream. Comparison of numerical results from both methods is performed. The results show that both methods can obtain important flow features and provide similar simulation in the wake of the turbine model. The actuator line method is able to give a better prediction in stream-wise velocity distribution, although it underestimates the turbulence intensity, circumferential velocity and vorticity magnitude slightly, compared with the full rotor geometry method. It is also found that the wake of the monopile and the rotor interact strongly in the downstream field, especially in the region immediately behind the structure. A strong interaction occurs within approximately two rotor diameters downstream.
\end{abstract}

Keywords: OpenFOAM; tidal stream turbine; actuator line method; full rotor geometry simulation; wake field

\section{Introduction}

There is an increasing demand for sustainable and environmentally-friendly energy resources throughout the world due to environmental pollution caused by continuous consumption of fossil fuel [1,2]. Tidal stream energy has been considered as one of the most promising clean energy resources. Compared with other kinds of renewable energy, such as wind energy and wave energy, tidal stream energy has the benefit of being highly predictable [3-5]. Tidal stream turbines (TSTs) are devices used to extract energy from a tidal stream, among which the horizontal-axis-type TSTs are mostly widely employed [6,7].

In order to make the best use of sea sites that are suitable for energy capture from tidal streams, multiple TSTs are commonly placed together as an array or a tidal farm [8]. If the array layout is well designed, much more energy could be extracted from a tidal stream [9-14]. For determination of each position of turbines in the array, the interaction between turbines in an array must be well considered. The performance of downstream turbines will decline if they are subjected to wake of 
upstream turbines [15]. Besides, a deeper insight into wake characteristics of a TST helps to better estimate its hydro-environmental effects, such as on water quality or sediment transportation [16,17].

In past decades, a number of studies have been carried out to understand the wake characteristics of a horizontal axis-type TST using both physical experiments and numerical simulations. In terms of physical experiments, Myers et al. [15] studied the early generation of marine stream energy converter arrays using multiple actuator disks to simulate TSTs. Their research highlighted an optimal lateral spacing between devices where, under certain conditions, flow could be accelerated between a pair of rotor disks. This enhanced flow speed gave rise to the counterintuitive notion of a downstream row of devices producing more power than the upstream row. Mycek et al. [18,19] investigated the ambient turbulence intensity (TI) effects on one single turbine and two in-line positioned turbines using the physical method. Their results showed that the wake properties were significantly affected by the upstream flow TI. The wake remained pronounced until 10 diameters downstream of the turbine with a TI of 3\%. However, the wake dissipated much faster and the upstream conditions, including velocity, TI and shear stress, were almost fully recovered at six diameters downstream with a TI of $15 \%$. A higher TI reduced the wake effects between turbines, and thus allowed a better comprise between inter-device spacing and individual performance. Chamorro et al. [20] performed laboratory experiments to study the dynamically strong interaction of a turbulent open channel flow with a bed-mounted axial-flow hydrokinetic turbine. They found that the flow velocity deficit increased monotonically with tip-speed ratio beyond four diameters downstream, and a complex non-monotonic behavior was observed in the vicinity of the turbine. The dominant effects of tip vortices within the first three rotor diameters at the turbine top-tip height were also demonstrated in [20]. Tedds et al. [21] conducted a detailed experimental investigation into the near wake of a model TST in a large-scale water channel facility. The results obtained by using the Reynolds-stress anisotropy tensor revealed the strongly anisotropic nature of the near-wake dynamics. Stallard et al. [22] investigated experimentally the mean wake of a TST in a wide flume. They found that the shear layers originating from the rotor tip circumference showed classic linear expansion downstream, and the shear layers expanded vertically in a rate 1.5 times of that horizontally. These shear layers merged by around two and half diameters downstream with a virtual origin at two diameters downstream of the rotor plane. Chen et al. [23] experimentally investigated the wake propagation behind a horizontal axis TST with three blades. The results indicated that the velocity reduction in the wake was caused by both the kinetic energy extraction and the blockage effects of the rotor and stanchion. The wake zone was found to shift towards the water surface, as a result of wake merging between turbine rotor and stanchion.

In recent years, computational fluid dynamics (CFD) simulations have gained increasing attention due to the rapid development of computer technology. Harrison et al. [24] used actuator discs (AD) in CFD models to predict the far wake of a horizontal axis TST. The results showed that the CFD model gave reasonable agreement with experimental results. However, the numerical simulation failed to reproduce precisely the turbulence level in the experiments. The AD method was further developed and used in $[25,26]$. Thiebot et al. [25] represented individual turbines in shallow water equation solvers based on the concept of AD. The simulation approach gave similar results to the bed friction method. Shives et al. [26] improved the AD method using adapted two-equation turbulence closures for the simulation of turbine wakes. Their improved model reduced the wake error and gave better prediction of power production of a turbine array, compared with the standard $k-\varepsilon$ model.

The blade element momentum (BEM) method can be considered as an enhanced AD method. Batten et al. [27] compared the results of the BEM simulation to experimental data measured in the wake of a scaled turbine. The comparison showed that the model was accurate and able to predict up to $94 \%$ of the variation in the experimental velocity data measured on the centerline of the wake. Edmunds et al. [28] revised the BEM method by modifying the angle of attack. The revised BEM method showed a significant improvement when predicting the performance of turbine as well as the $\mathrm{TI}$ in the wake region. 
Compared with the full rotor geometry (FRG) method, in which the rotor blade shape is fully presented in the model, the AD and BEM method cannot predict the detailed flow features caused by the finite number of blades [29]. The FRG method can also more accurately predict the hydrodynamic loads acting on a TST and its wake properties [30]. Nuernberg et al. [31] used the FRG method to investigate the wake field in a tidal array with four turbines, and their results showed a good agreement between simulations and experiments. Further insight into the flow field within an array was also provided. Chen et al. [32] investigated flow field between a TST and the seabed. They found that the axial flow velocity between the downward facing blade and seabed was accelerated, and the seabed boundary layer profile was therefor changed. However, in order to use the FRG method, a large number of mesh elements were necessary to describe the turbine rotor shape precisely. In addition, the FRG method needs to handle a moving grid in order to account for the movement of rotor in the computational domain. The grid elements inside the rotating zone are moving together with the rotor to represent the actual rotation effect. Liu et al. [30] calculated the wake of a TST with 32 cores on the high-performance computing (HPC) cluster with 32 GB random access memory (RAM) per server using the FRG method, and it cost approximately one week. Therefore, the implementation of the FRG method is computationally expensive.

The actuator line (AL) method can be considered as a combination of the BEM and FRG methods. It adds forces induced by turbine blades to the flow field as sink terms like the BEM method, and the sink terms also rotate against time like rotor rotation in the FRG method. Baba-Ahmadi et al. [33] studied the wake of a laboratory-scale TST using the AL method in combination with the large eddy simulation (LES) technique, which cost $6200 \mathrm{cpu}$-hours in a HPC cluster. The results showed a reasonable agreement with experimental data. However, some discrepancies occurred due to failure in the simulation of the turbulence generated in the boundary layer and from the blade vibration. Baratchi et al. [34] also used the AL method coupled with LES to simulate a TST in straight and yawed flows. Their results showed that the AL method was capable of capturing wake unsteadiness and tip and root vortices in the wake. The advantage of the AL method over the BEM method was also highlighted in [34]. Compared with the FRG method, Bachant [35] found that the AL method could provide four orders of magnitude reduction in computational expense when using the Reynolds-averaged Navier-Stokes (RANS) with turbulence model.

This study presents the investigation into wake characteristics of a TST supported by a monopile using both the AL method and FRG methods. The numerical simulation is conducted using the open source software OpenFOAM [36,37]. A $k-\omega$ shear stress transport (SST) turbulent model [31] is adopted in this study. Validations against experimental results are performed to ensure the accuracy and independence of the numerical results. Then, differences of these two methods in simulating mean wake characteristics of the TST are analyzed, including mean velocity distribution, TI and vorticity. The interaction between wakes of the monopile and turbine rotor are also analyzed. The study explores the capability of the AL method and FRG method to simulate the wake features with a monopile.

\section{Methods}

\subsection{Experimental Setup}

The experiment is carried out in a recirculating flume with a length of $50 \mathrm{~m}$ and a width of $1.2 \mathrm{~m}$. Water flows through a honeycomb straightener to damp the rotational flow component induced in the inlet. A uniform flow is obtained before the flow reaches the turbine rotor. The device is placed in the middle of the flume with $25 \mathrm{~m}$ downstream away from the inlet (See Figure 1). An acoustic doppler velocimeter (ADV, Nortek AS, Rud, Norway) is used to measure the flow field with a sampling rate of $100 \mathrm{~Hz}$. Throughout the measurements, the signal to noise ratio (SNR) is maintained above 18. The collected data are filtered by removing spikes using the phase-space thresholding method [38] when data are with low correlation $(<70 \%)$. 


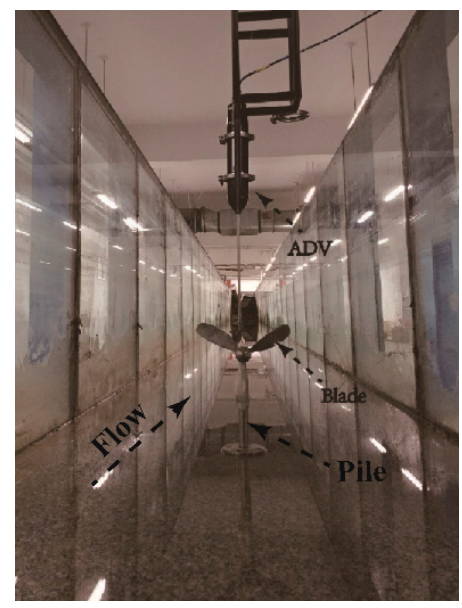

Figure 1. The position of the model turbine in the flume.

The turbine rotor used in the experiment has a diameter of $0.27 \mathrm{~m}(D)$. The length of nacelle connected to the rotor is about $0.216 \mathrm{~m}(\approx 0.8 D)$ and the supporting pile has a diameter of $0.027 \mathrm{~m}$. The foil of rotor blades is chosen from those foil shapes designed by the National Advisory Committee for Aeronautics (NACA), as detailed in Table 1. The blades are constructed of aluminum and fixed to a hub with a diameter of $0.04 \mathrm{~m}$.

Table 1. Blade geometry.

\begin{tabular}{cccccc}
\hline $\mathbf{r} / \mathbf{R}$ & $\mathbf{c} / \mathbf{R}$ & Twist (deg) & $\mathbf{r} / \mathbf{R}$ & $\mathbf{c} / \mathbf{R}$ & Twist (deg) \\
\hline 0.05 & 0.33 & 47.94 & 0.55 & 0.61 & 27.79 \\
0.10 & 0.33 & 47.94 & 0.60 & 0.61 & 26.05 \\
0.15 & 0.33 & 45.81 & 0.65 & 0.61 & 24.36 \\
0.20 & 0.36 & 43.68 & 0.70 & 0.60 & 22.69 \\
0.25 & 0.43 & 40.78 & 0.75 & 0.58 & 21.01 \\
0.30 & 0.51 & 37.25 & 0.80 & 0.57 & 19.29 \\
0.35 & 0.55 & 35.26 & 0.85 & 0.53 & 17.47 \\
0.40 & 0.58 & 33.31 & 0.90 & 0.49 & 15.43 \\
0.45 & 0.60 & 31.41 & 0.95 & 0.42 & 12.91 \\
0.50 & 0.61 & 29.57 & 1.00 & 0.16 & 9.50
\end{tabular}

Note: $\mathrm{R}$ is the rotor radius, $\mathrm{r}$ is radial location of local blade cross-sections, $\mathrm{c}$ is chord length of local blade cross-sections.

Throughout the experiment, the mean approach velocity is maintained at $0.35 \mathrm{~m} / \mathrm{s}$ and the water depth (h) remains as $0.54 \mathrm{~m}(=2 D)$. The distance between the flume bottom and the hub center is $0.27 \mathrm{~m}(=1 D)$. The turbine rotor rotates at a speed of $90 \mathrm{rpm}$, which gives a tip speed ratio (TSR) of 3.64. Flow field within the downstream distance of $1 D-8 D$ is measured using the ADV, as shown in Figure 2 .



Figure 2. Measurement locations in the downstream field. The dashed lines indicate the cross-sections where velocity measurement was made along $y$ direction and the red spots indicate the measurement positions along $x$ direction. 


\subsection{The Actuator Line (AL) Model}

The AL method is based on the blade element theory combined with a Navier-Stokes description of the flow field. The method is implemented using an extension library, turbinesFoam [35], which is developed in OpenFOAM (v6.0) (the OpenFOAM Foundaiton, London, UK). A brief introduction is given here for completeness.

In the AL method, the governing equations are the conservation of mass and momentum and these are discretized using the finite volume method. The time-averaged forms of these equations are:

$$
\begin{gathered}
\frac{\partial \overline{u_{i}}}{\partial x_{i}}=0 \\
\frac{\partial \overline{u_{i}}}{\partial t}+\frac{\partial \overline{u_{i} u_{j}}}{\partial x_{j}}=-\frac{1}{\rho} \frac{\partial \bar{p}}{\partial x_{i}}+v \frac{\partial^{2} \overline{u_{i}}}{\partial x_{j}^{2}}+v_{t} \frac{\partial \tau_{i j}}{\partial x_{j}}+\overline{f_{i}}
\end{gathered}
$$

where $f_{i}$ is the source term in the $i$ direction due to the blades' forces.

In the AL method, these source terms are calculated based on the blade element theory, with the turbine blade being divided into several blade elements. In each element, the tabulated 2-D airfoil characteristics are used to determine loads on the rotor blades. Figure 3 shows a cross-sectional airfoil element at radius $r$ in the $(Z, \theta)$ plane.

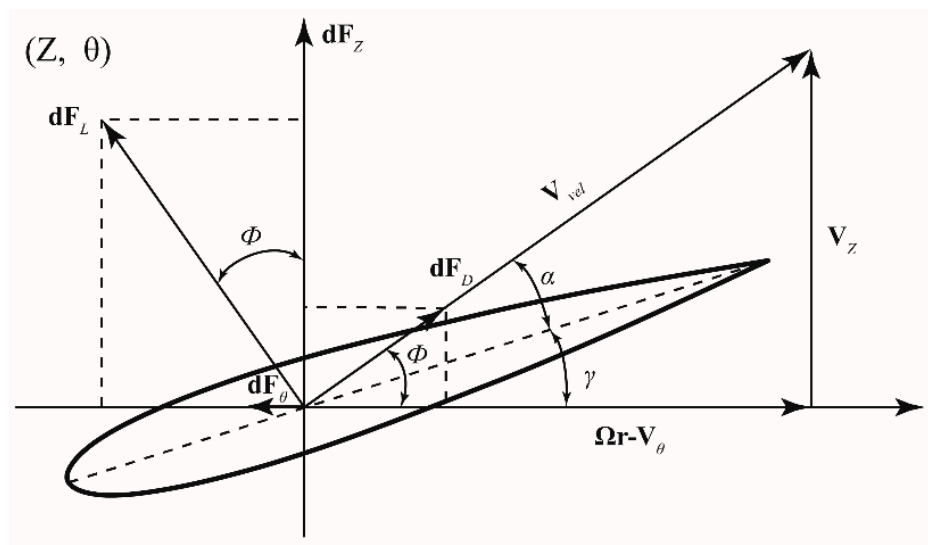

Figure 3. Cross-sectional airfoil element showing velocity and force vectors.

As shown in Figure 3, the axial and rotational forces by the flow on each section are calculated by,

$$
\begin{aligned}
& \mathrm{d} F_{Z}=1 / 2 \rho V_{v e l}^{2} c d r\left(c_{l} \cos \phi+c_{d} \sin \phi\right) \\
& \mathrm{d} F_{\theta}=1 / 2 \rho V_{v e l}^{2} c d r\left(c_{l} \sin \phi-c_{d} \cos \phi\right)
\end{aligned}
$$

in which $\rho$ is the water density, $V_{v e l}$ is the relative velocity, $d r$ is the width of blade element, $c_{l}$ and $c_{d}$ are the lift coefficient and drag coefficient, respectively, dependent on sectional Reynolds number $R_{e}$ and local angle of attack $\alpha$.

The relative velocity $V_{v e l}$ is calculated by,

$$
V_{v e l}=\sqrt{V_{Z}^{2}+\left(\omega r-V_{\theta}\right)^{2}}
$$

in which $V_{Z}, V_{\theta}$ are the flow velocity in the $Z$ and $\theta$ direction, respectively. $\omega$ is the blade's rotation speed.

Flow angle $\phi$ in Equations (3) and (4) is calculated by,

$$
\tan \phi=\frac{V_{Z}}{\omega r-V_{\theta}}
$$


The angle of attack $\alpha$ necessary for $c_{l}$ and $c_{d}$ is calculated from $=\phi-\gamma$, where $\gamma$ is the blade element's twist angle.

Then the calculated force at each AL element is smoothly projected to the surrounding region in order to avoid instability due to steep gradients. For this purpose, the 3-D Gaussian projection function has been implemented. The width of this projection $\eta$ is controlled by a single parameter $\epsilon$, which is then multiplied by the AL element force and imparted on a cell with distance $|\vec{r}|$ from the AL element quarter chord location. For further details, please refer to [35].

Figure 4 shows the distribution of source terms obtained from the AL method using the 3-D projection. As can be seen, the distribution of the source terms in the AL method resembles the actual geometry of a rotor. The asymmetrical distribution of source terms is induced by the bottom boundary layer. When the blade is facing upward, it is exposed to larger approach velocity and resultantly, larger source terms are added to the governing equations.

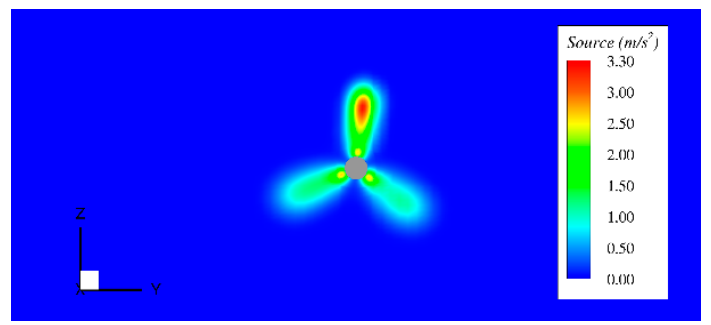

Figure 4. Source terms implemented in the actuator line (AL) method.

Figure 5 shows the computational domain for the AL method. The geometry model includes the nacelle and mono-pile foundation. The size of the computational domain is about $8.1 \times 1.2 \times 0.54 \mathrm{~m}$. It had an inner domain having the disc located at the coordinate system's origin with radius $\mathrm{R}$ equal to the model turbine. The computational domain also had an outer domain which represented the flow field in the tank with dimensions of $5 D$ in the upstream direction and $25 D$ in the downstream direction.

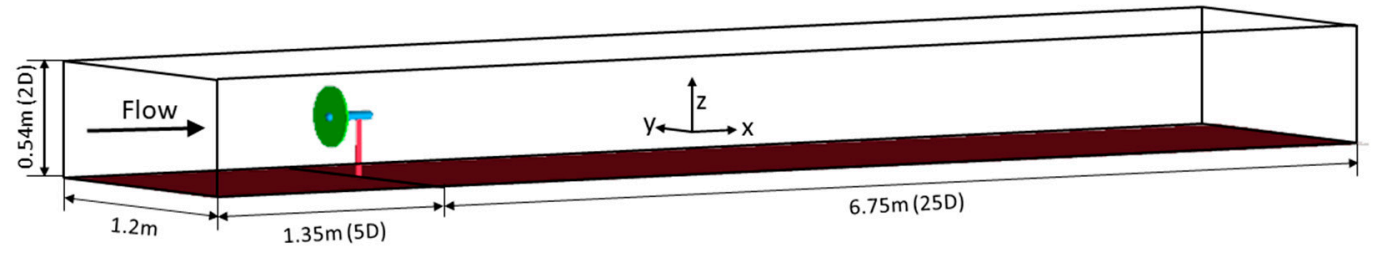

Figure 5. The computational domain for the AL method. The geometry in blue is the nacelle and the one in red is the mono-pile foundation.

For boundary conditions, the vertical profile of $u$ is determined from the measured vertical profile of $u$ (see Figure 6 ) at the inlet. The profile of $u$ is assumed in the form of $1 / 7$ th law, as follows,

$$
\begin{aligned}
u(z)= & U(z /(0.19 h))^{1 / 7}, \mathrm{z}<0.22 \mathrm{~m} \\
& u(z)=U, \mathrm{z}>0.22 \mathrm{~m}
\end{aligned}
$$

where $U$ is the mean approach velocity and estimated as $0.35 \mathrm{~m} / \mathrm{s}$. At the inlet, The TI level is set as $5 \%$ based on measurement results in Figure 6. Zero gradient boundary is specified at the outlet and no slip conditions are imposed at both the bottom and lateral walls. In order to save computational cost, a symmetry condition is applied at the top to ignore the free surface. 

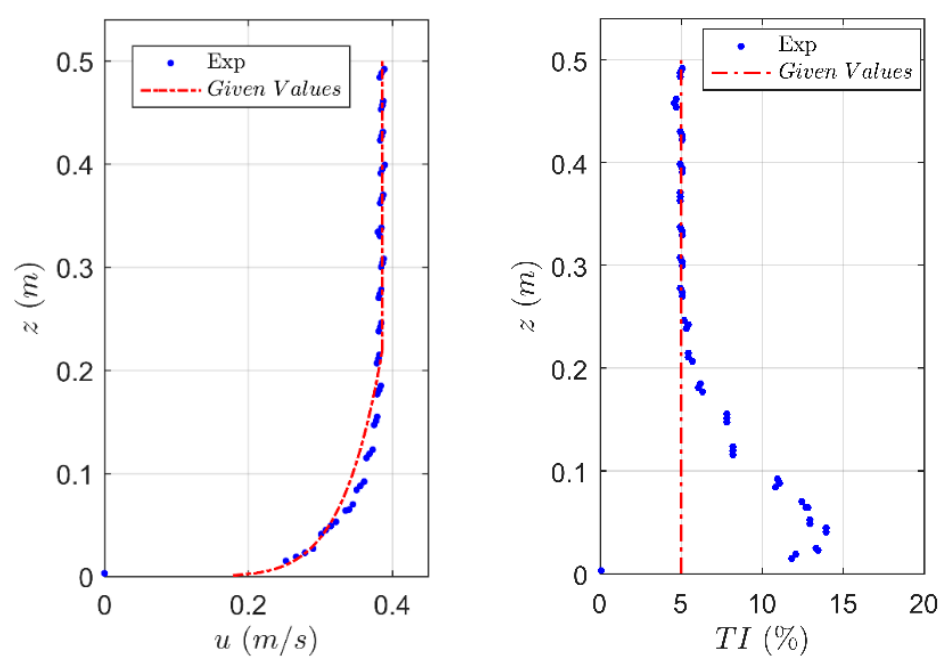

Figure 6. Vertical profiles of $u$ and turbulence intensity (TI) at the inlet boundary.

\subsection{The Full Rotor Geometry (FRG) Method}

The governing equation for the FRG method is the same with Equations (1) and (2) but with the source term $f_{i}$ omitted. The sliding-interface technique is used for its implementation in OpenFOAM [39]. Figure 7 shows the mesh around the turbine. The whole computational domain is divided into the static zone and the rotational zone. In the simulation, the rotational region rotates constantly to account for the movement of the three-bladed tidal turbine at constant TSR, here set as 3.67 as measured in the experiment. The momentum exchange between the rotational zone and the static zone is achieved by using the arbitrary mesh interface (AMI). The data calculated along the interface are transferred by interpolation of the flux on the adjacent cell elements. In the rotational zone, an unstructured mesh with prisms is used so that the geometry of the rotor could be accurately captured to improve accuracy of the numerical results. However, the static zone is discretized using a structured mesh to reduce the number of mesh elements. No slip wall conditions are imposed at the pile and nacelle, and a rotating wall condition is applied to blade surfaces. In addition, standard wall functions for turbulence models are used to bridge the blade walls and fully turbulence region to save computation time and resources. For non-dimensional wall distance $\mathrm{y}^{+}$of grid cells adjacent to blades, $\mathrm{y}^{+}$of the first layer prism is within the range of $30<\mathrm{y}^{+}<300$ to accurately capture the turbulent flow near the blade surfaces. Other set-up details for the FRG method are the same as those in the AL method.

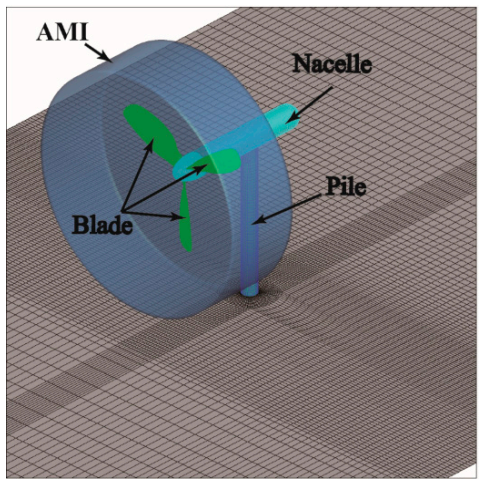

(a)

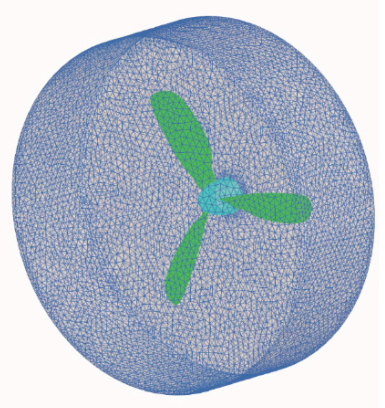

(b)

Figure 7. Implementation of the full rotor geometry (FRG) method in OpenFOAM. (a) Flow field is divided into two regions, i.e., static region and rotational region. (b) The rotational region with unstructured mesh. 


\subsection{Mesh Refinement Study}

For the AL method, a structured hexahedral mesh is generated. Figure 8 shows the cut plane of the mesh across the rotor center. The mesh is refined around the disc domain, specifically in the radial direction within the disc. Besides, a relatively high mesh resolution is used near the disc just upstream and downstream the disc. For the FRG method, it has a rotational inner zone with an unstructured tetrahedral mesh adapted to the rotor blade geometry and a stationary outer zone with a structured hexahedral mesh. Three cases with coarse, medium and fine meshes are also conducted. Table 2 shows the three mesh densities for both the AL method and the FRG method. Their results are compared with the measured velocity distribution from physical experiments, as shown in Figure 9. The comparisons between different mesh densities are shown in Table 3. For both methods, cases with a higher mesh density obtain better simulation results, as expected. However, the difference between medium and fine meshes is negligible. Therefore, medium meshes are then used for both methods. The AL method has a total number of 2.39 million elements with the finest cell size nearly $\mathrm{R} / 30$ in the disc domain and the FRG method has a total number of 5.04 million elements with 1.35 million elements inside the rotational inner zone. The maximum size for surface mesh on the blade surfaces is specified as $0.001 \mathrm{~m}$ for the FRG method. Both the simulations are computed on a workstation with 16 Intel ${ }^{\circledR}$ Xeon $^{\circledR}$ processors and 32 GB RAM (Microsoft, Redmond, WA, US). The calculation duration is chosen to be $30 \mathrm{~s}$ so that the approaching flow can go through the whole computational domain. The AL method takes about 2 days to finish the simulation while the FRG simulation takes about one week. The FRG method requires much more computing time mainly for the following reasons: (1) it has a much larger size of mesh, (2) it needs to handle a dynamic mesh, which accounts for the rotation of rotor blades, and (3) it needs to calculate the flux exchange between the rotational zone and stationary zone.



Figure 8. Mesh generation in the rotor plane for the AL method.

Table 2. Mesh size for the AL method and FRG method (million).

\begin{tabular}{ccc}
\hline Mesh Density & AL Method & FRG Method \\
\hline Coarse & 1.51 & 2.45 \\
Medium & 2.39 & 5.04 \\
Fine & 4.26 & 8.21 \\
\hline
\end{tabular}




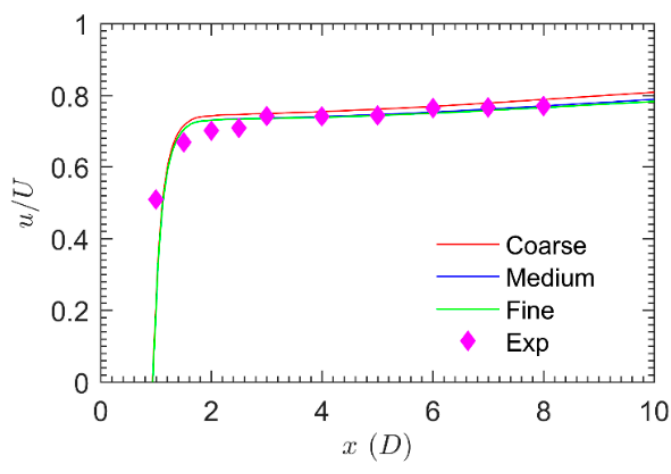

(a)

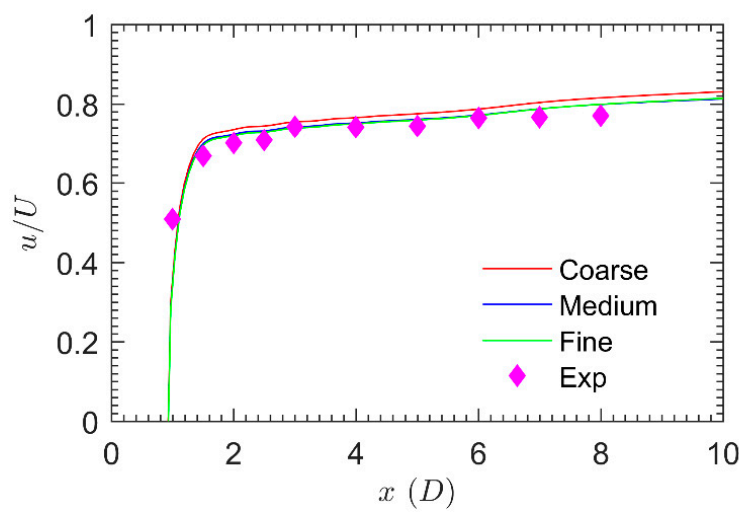

(b)

Figure 9. Stream-wise velocity distribution along the rotational axis with various mesh density. (a) Mesh refinement study for the AL method. (b) Mesh refinement study for the FRG method.

Table 3. Maximum variation of stream-wise velocity along rotational axis.

\begin{tabular}{ccc}
\hline Mesh Density & AL Method & FRG Method \\
\hline Coarse & $5.1 \%$ & $4.4 \%$ \\
Medium & $0.2 \%$ & $1.3 \%$ \\
Fine & - & - \\
\hline
\end{tabular}

For both the AL and FRG method, time integration is carried out by the Euler scheme and the equation set is solved using the Pressure-Implicit with Splitting of Operators (PISO) algorithm. The maximum time step is set as $0.002 \mathrm{~s}$ and the Courant-Friedrichs-Lewy (CFL) condition is determined by setting the maximum Courant number less than 1 during computation.

\section{Results}

\subsection{Velocity Distribution}

\subsubsection{Mean Velocity Distribution}

Figure 10 shows velocity recovery along the rotational axis using both the AL method and FRG method. Both methods give a similar trend with the experimental results, although some discrepancies occur in the near region from $x=1 D$ to $2.5 D$, which could be due to the free surface treatment. Flow velocity recovers to around $0.72 U$ by $2 D$ downstream and, afterwards, the recovery rate slows down remarkably, reaching about $0.80 \mathrm{U}$ until $10 \mathrm{D}$ downstream. The difference between these two methods occurs at $x=4 D$, after which the FRG method results in a slightly faster recovery rate. This difference may be due to the higher turbulence level in the downstream field obtained in the FRG method, which is shown in Section 3.2. The higher turbulence level intensifies momentum exchange between the wake field and the ambient free stream, therefore resulting in a faster wake recovery [19]. Because of this, the FRG method tends to overestimate the flow recovery after $x=4 D$. The AL method has a better agreement with experimental data with a root mean square error (RMSE) $(\sim 0.018)$ slightly lower than that of the FRG method ( 0.021). 


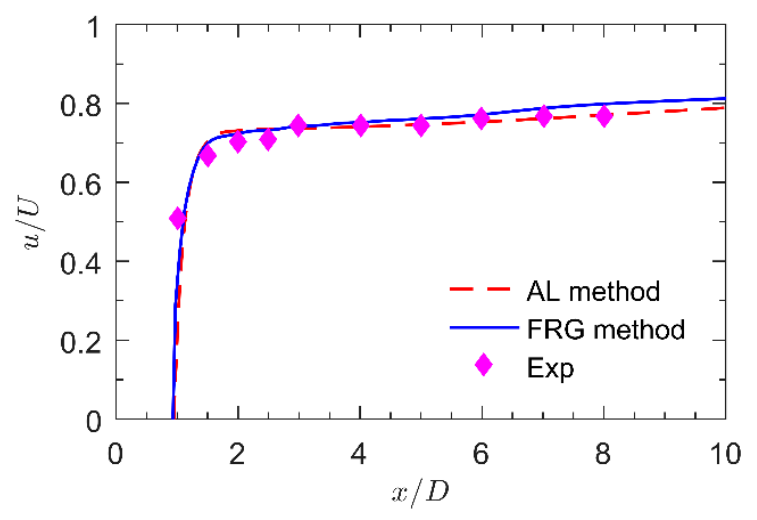

Figure 10. Velocity recovery along the rotational axis.

Figure 11 shows the stream-wise velocity distribution in the transverse direction in the downstream field from $x=1 D$ to $8 D$. The plots show a reasonable agreement with experimental data for the numerical results obtained by both methods, especially in the far wake region beyond $x=5 D$, where Gaussian distribution applies [40]. In the near wake region from $x=1 D$ to $4 D$, there are some discrepancies around the wake center. These discrepancies may be due to the following reasons: (1) the free surface is ignored in this study, and (2) the end of nacelle is approximately described as a hemispheroid in numerical simulation, which is an ellipsoid in reality. It can also be seen that velocity distribution is asymmetry in the wake. This feature is reflected to some extent in the FRG method. However, the stream-wise velocity is totally symmetrical in the across direction obtained by the AL method.

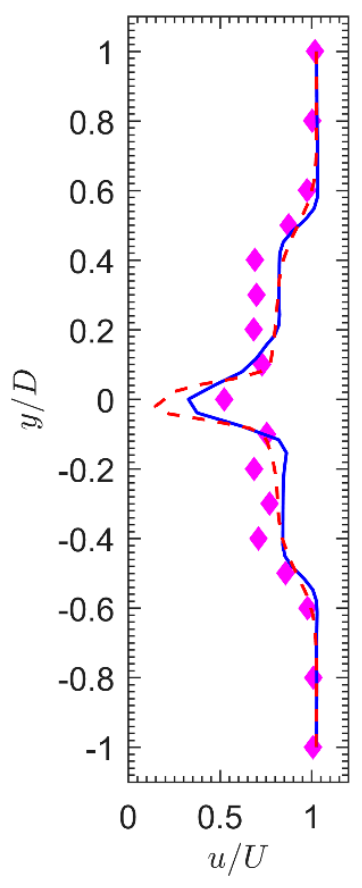

(a)

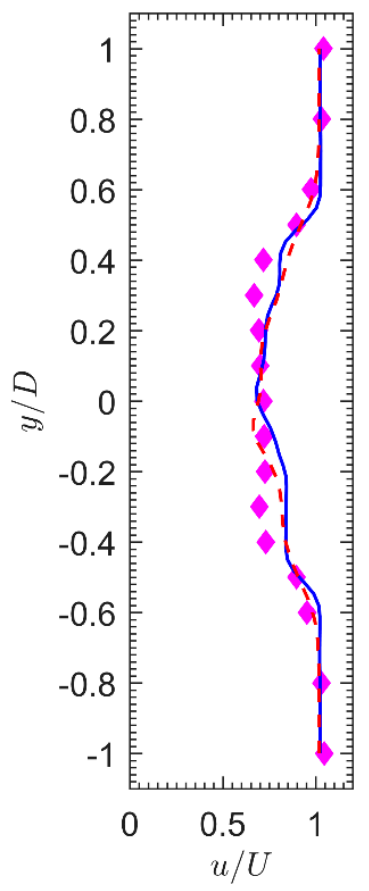

(b)

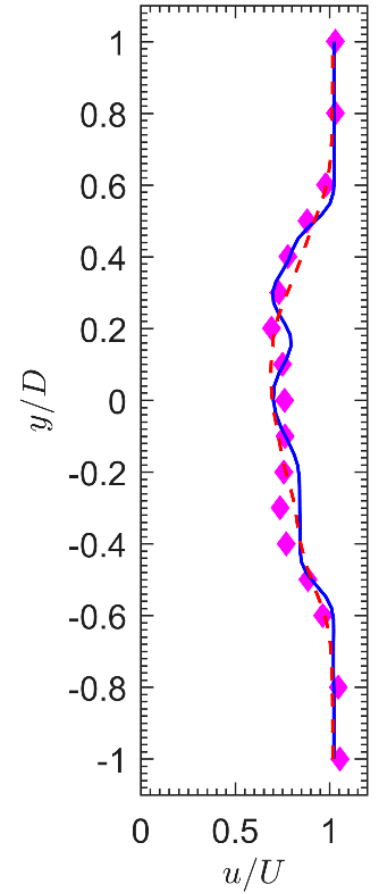

(c)

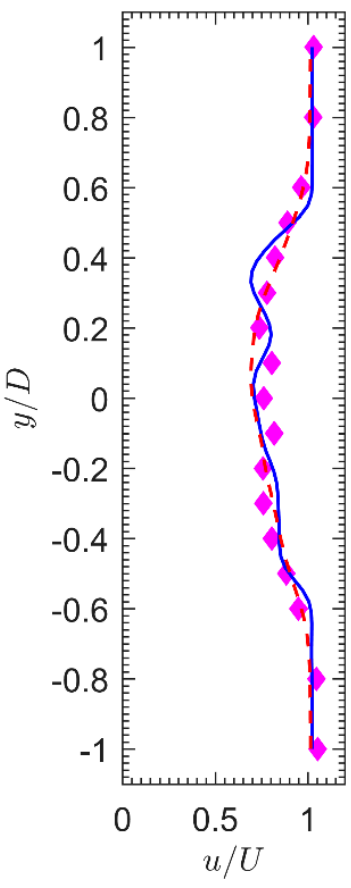

(d)

Figure 11. Cont. 




(e)

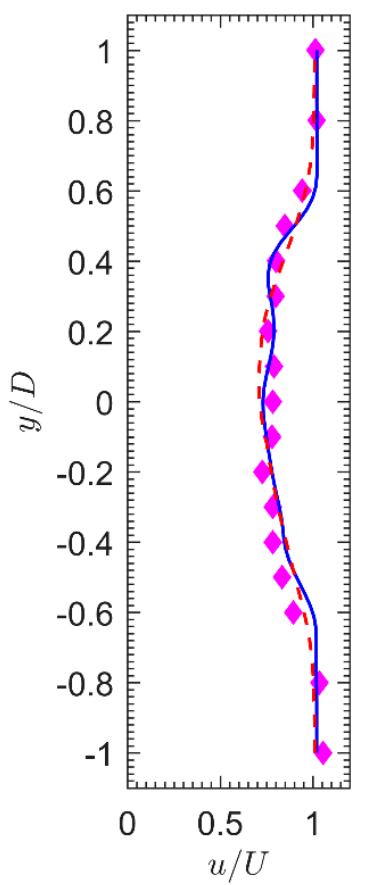

(f)

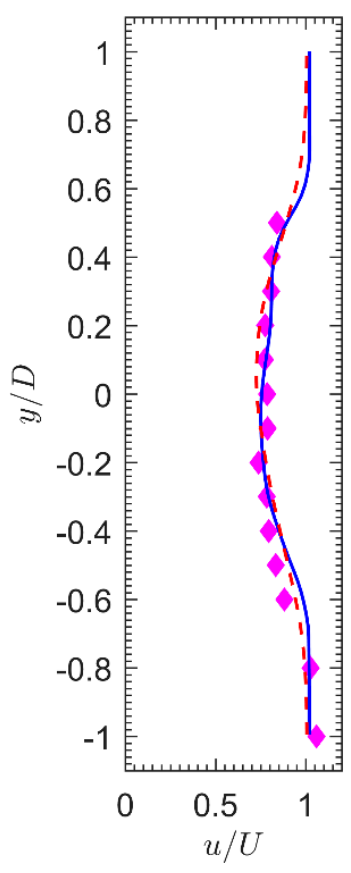

(g)

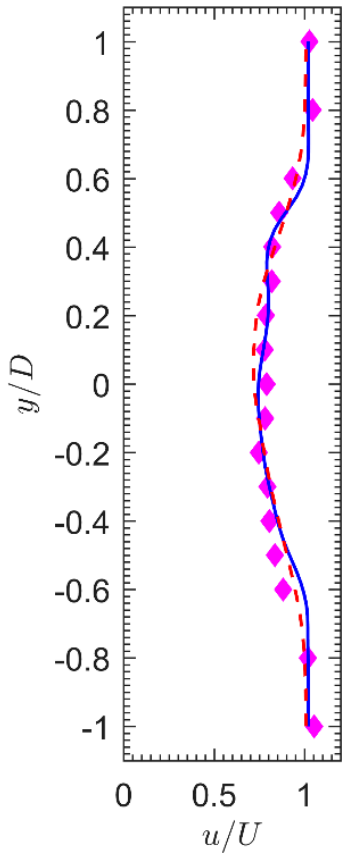

(h)

Figure 11. Stream-wise velocity distribution in the transverse direction. $(\mathbf{a}) \mathrm{x}=1 D .(\mathbf{b}) \mathrm{x}=2 D .(\mathbf{c}) \mathrm{x}=3 D$. (d) $x=4 D$. (e) $x=5 D$. (f) $x=6 D$. (g) $x=7 D$. (h) $x=8 D$. Dashed line: the AL method. Solid line: the FRG method. Diamond point: Exp.

The RMSE of normalized stream-wise velocity $(u / U)$ along the transverse direction for both methods is estimated at various distances downstream, as shown in Figure 12. This shows that the AL method generally gives a better prediction of velocity distribution, especially from $x=2 D$ to $6 D$. After $x=6 D$, these two methods show the same performance in predicting velocity distribution along the transverse direction overall.

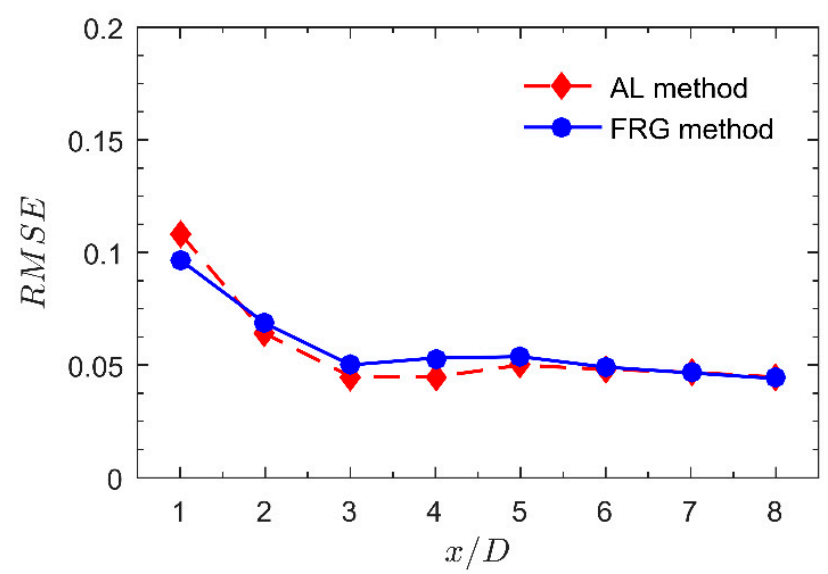

Figure 12. Root mean square error (RMSE) of velocity prediction at various distance downstream.

\subsubsection{Instantaneous Velocity Distribution}

Figure 13 shows instantaneous velocity distribution in the horizontal plane across the hub center. The effect of the swirl induced by the turbine blade rotation can be observed clearly using both methods, in particular within $1 D$ downstream. It can also be seen that wake of the nacelle interacts strongly with the turbine's wake until $2 D$ downstream. This interaction might interfere with the swirl motion of the turbine's wake, thereby affecting its flow structures. As shown in Figure 13, the turbine's 
wake has a slightly larger velocity magnitude in the AL simulation, compared with that in the FRG simulation. This could be due to the fact that in the FRG simulation, flow stops when meeting the rotor blades, but in the AL simulation it passes through the space directly where rotor blades shall occupy. Correspondingly, the FRG method has a larger velocity prediction around the blade tips as a result of mass conservation (see the rectangular region in Figure 13b).

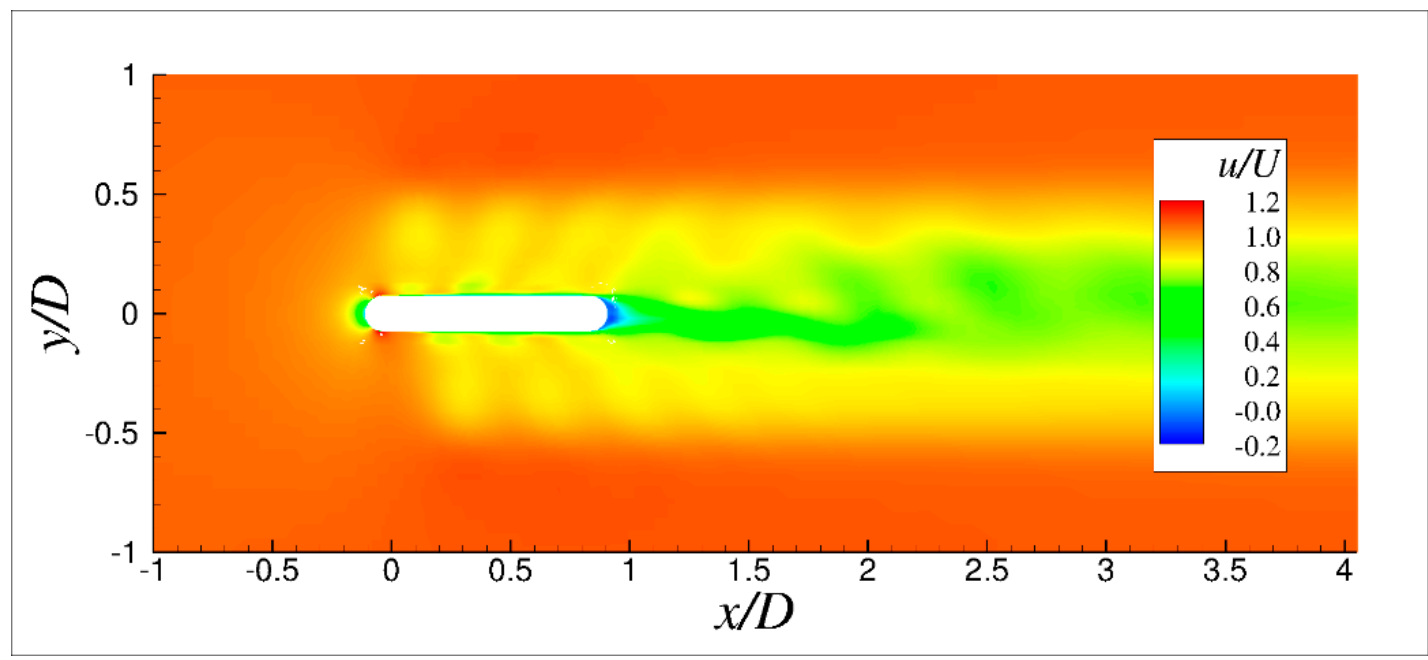

(a)

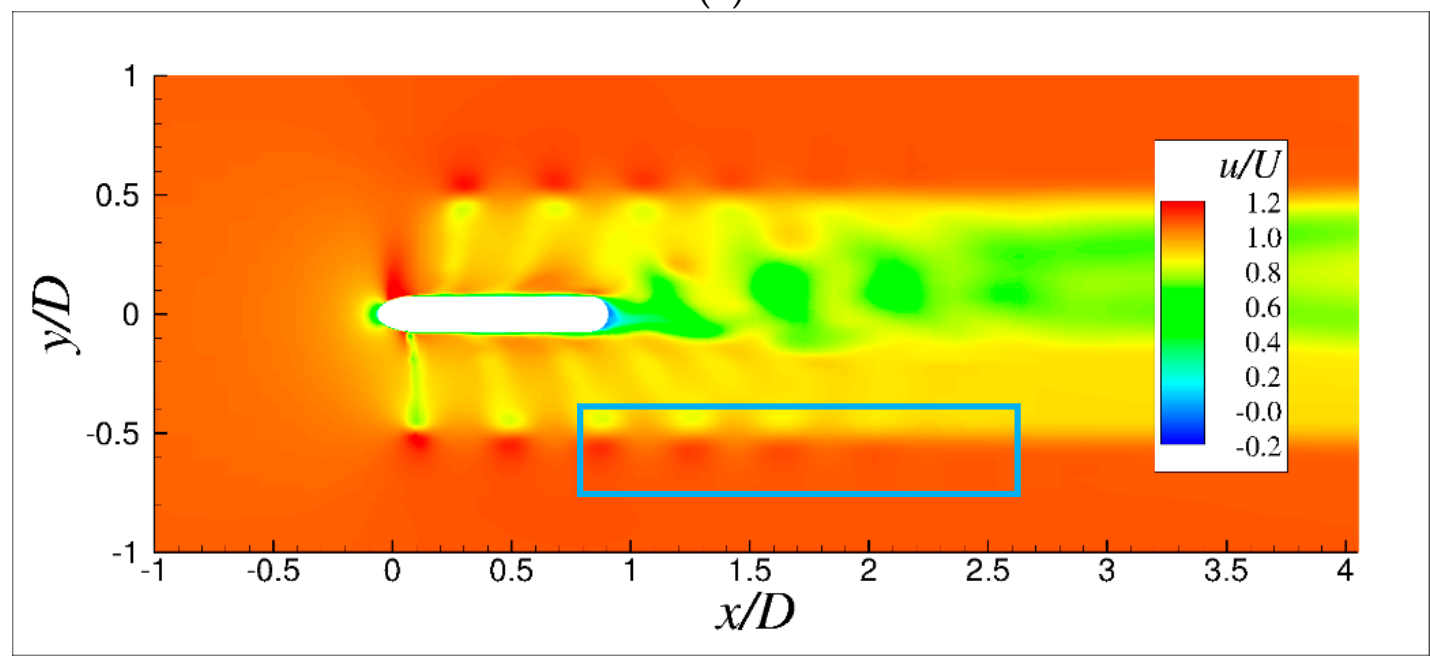

(b)

Figure 13. Instantaneous velocity distribution in the horizontal plane across the hub center. (a) The AL method. (b) The FRG method.

Figure 14 shows instantaneous velocity distribution in the vertical plane across the hub center. The swirl motion can be observed clearly above the hub height within $1 D$ downstream. However, the swirl disappears beneath the hub due to the presence of the monopile. Instead, a low velocity region occurs behind the monopile with a larger range simulated by the AL method. This might be due to the higher turbulence level predicted by the FRG method, which will be shown in Section 3.2. 


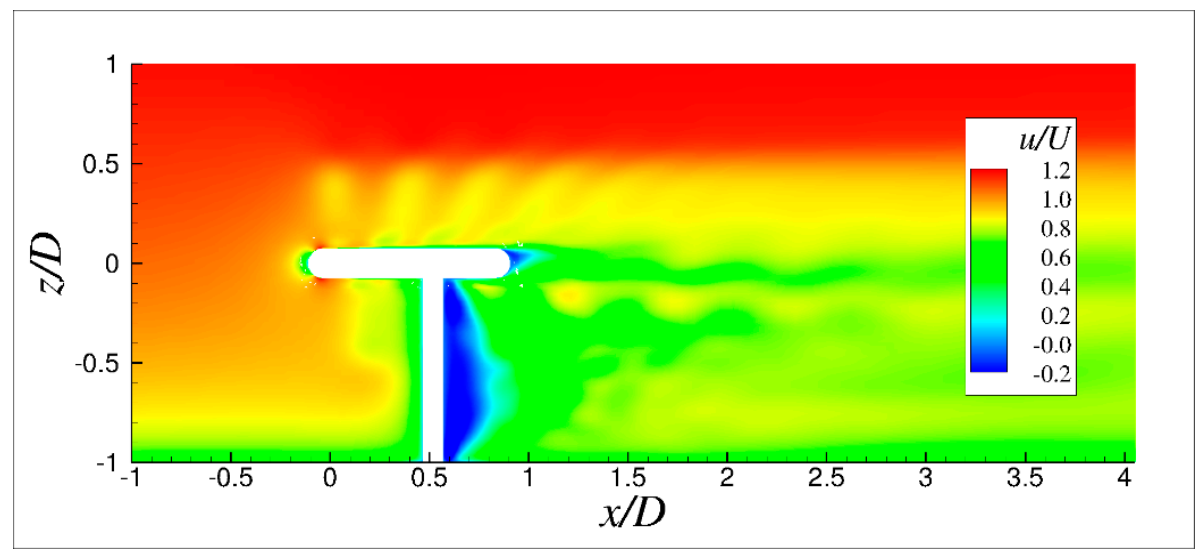

(a)

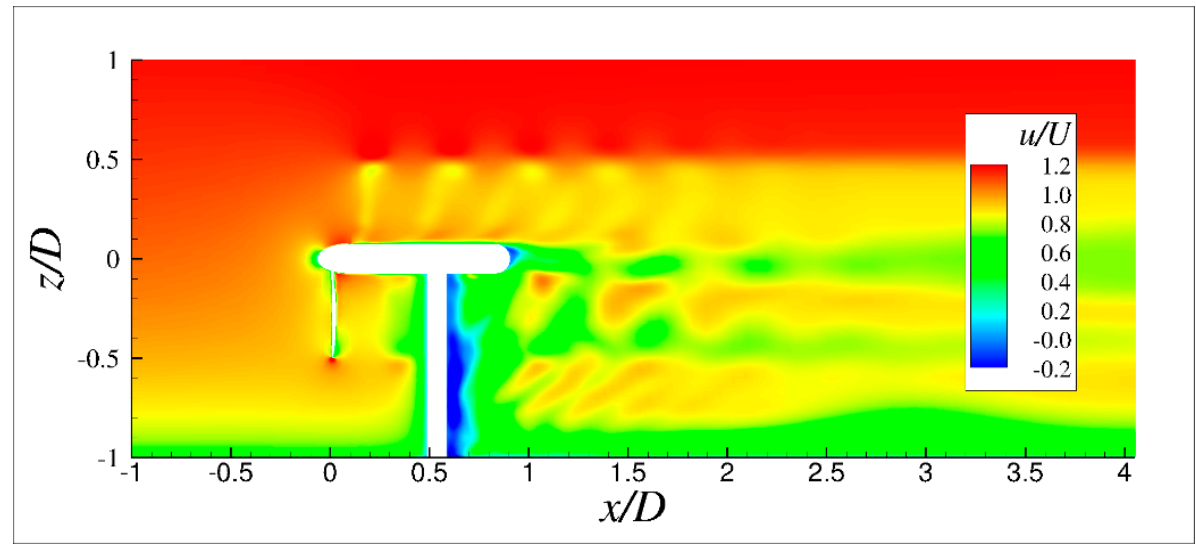

(b)

Figure 14. Instantaneous velocity distribution in the vertical plane across the hub center. (a) The AL method. (b) The FRG method.

Circumferential velocity distribution at multiple distances downstream obtained using both methods is shown in Figure 15. The two methods share a similar pattern in simulating the circumferential velocity. Large anticlockwise circumferential velocity due to the effect of the swirl induced by the rotation of the turbine's blades can be observed at $\mathrm{x}=1 D$, which corresponds to the clockwise rotation of the blades as a result of momentum conservation. Its magnitude drops quickly from $\mathrm{x}=1 D$ to $4 D$. The largest circumferential velocity is found near the wake center at different distances downstream. Within the swept area, there is a region with relatively lower stream-wise velocities due to the shadow effects of the supporting nacelle and monopile. Compared with the FRG method, the AL method has a lower estimation of circumferential velocity. It is still remarkable until $\mathrm{x}=4 D$ is observed from the FRG method, however, it is negligible at $\mathrm{x}=4 D$ in the AL method. The difference is due to the fact that the relative motion of rotor geometry and flow field is not fully resolved by the AL method. 


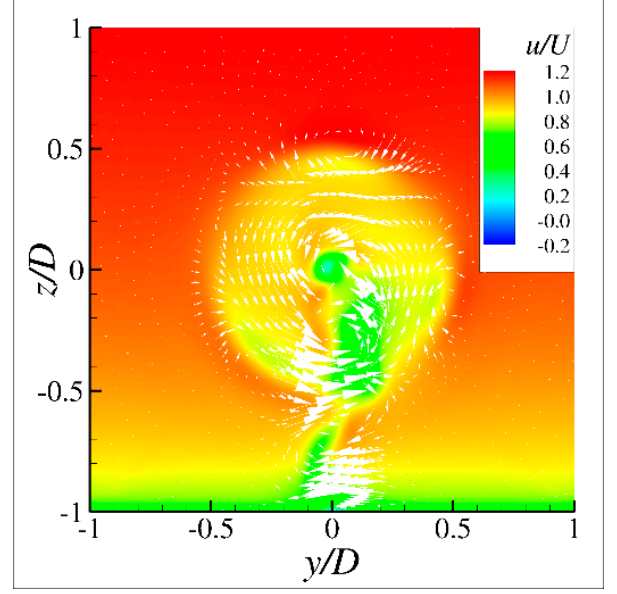

(a)

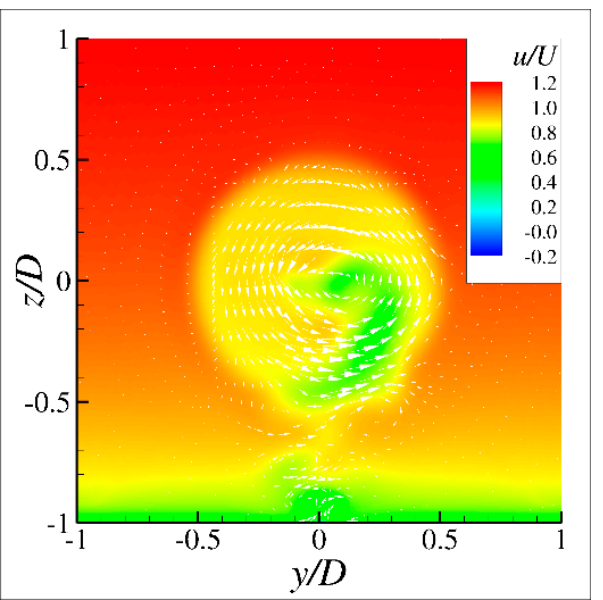

(b)



(c)

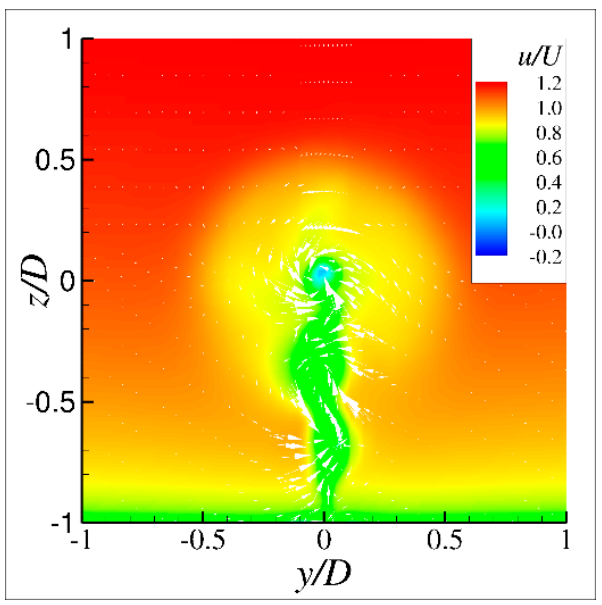

(d)

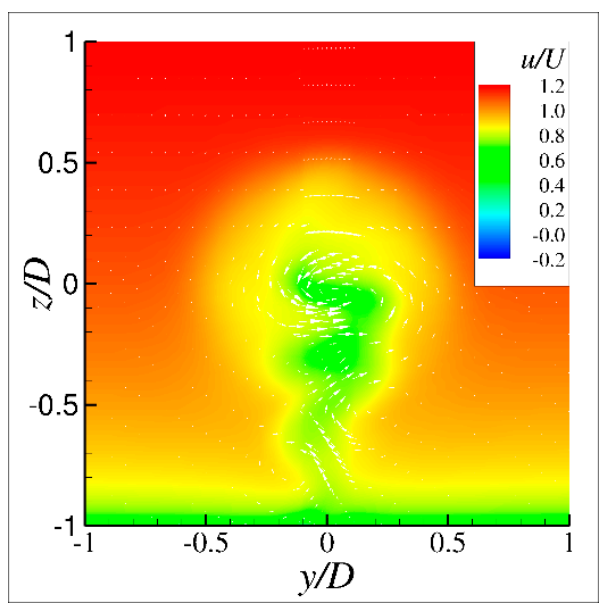

(e)

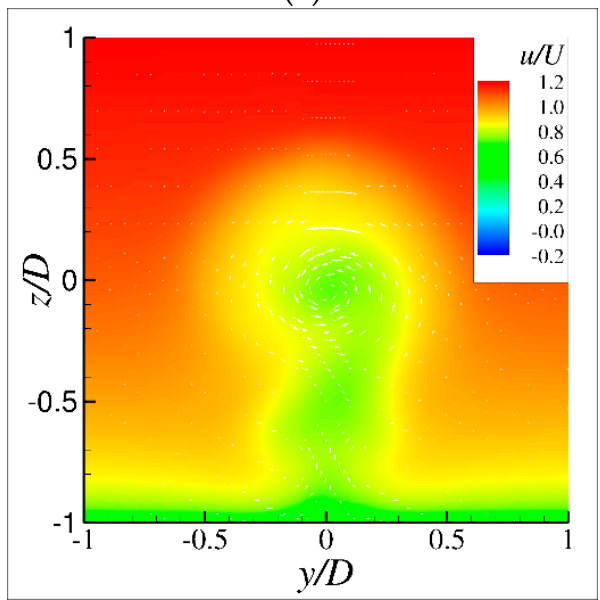

(f)

Figure 15. Development of wake rotation in the downstream with the color presenting the stream-wise velocity and the arrowhead circulation vector. (a-c) The FRG method for $\mathrm{x}=1 D, 2 D, 4 D$, respectively. (d-f) The AL method for $\mathrm{x}=1 D, 2 D, 4 D$, respectively.

\subsection{Turbulence Intensity (TI)}

Figure 16 shows TI calculated using both methods. These two methods share the same pattern of the TI in the wake, with larger TI in the center. The largest value is found right behind the nacelle in the central and it gradually decreases as water flows downstream. Compared with the FRG method, the AL method obtains a lower TI level. The main reason is that turbulence generated in the blade 
boundary layer and fed to the flow is ignored by the AL method [33]. The other difference is that the FRG method obtains two peak TI values in downstream along the blade tip, however it is not reflected in the AL method. This means that the AL method ignores tip vortices as well. The two peak values in the FRG method decrease from $x=1 D$ and become negligible at $x=2 D$.

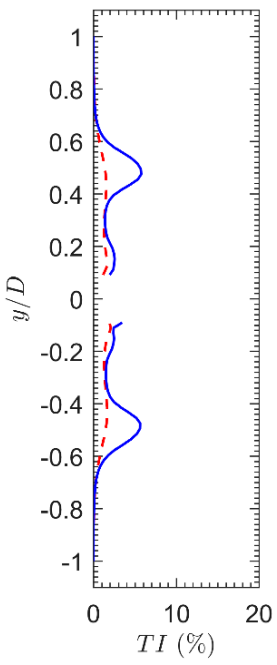

(a)

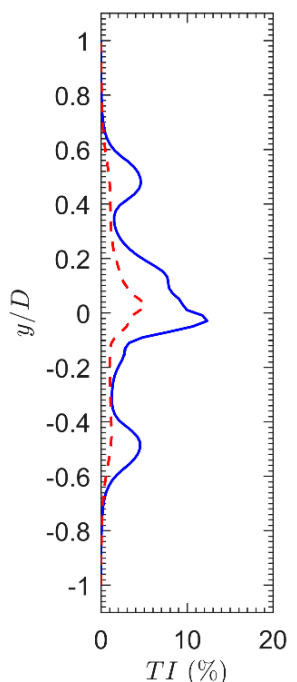

(b)

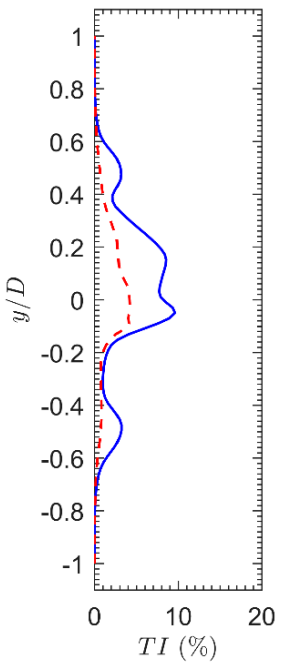

(c)

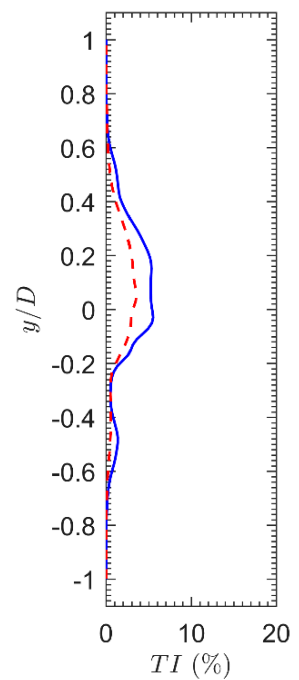

(d)

Figure 16. Turbulence intensity (TI) level calculated using both methods in the transverse direction from $x=0.5 D$ to $2 D$. (a) $x=0.5 D$. (b) $x=1 D$. (c) $x=1.5 D$. (d) $x=2 D$. Dashed line: the AL method. Solid line: the FRG method.

Figure 17 shows TI level from bottom to water surface at multiple distances downstream. Both methods show large TI level beneath the nacelle from $x=1 D$ to $2 D$. This high level of turbulence is induced by the interaction between wakes of the rotor and the monopile. However, the TI level drops quickly. It is close to $20 \%$ at $x=1 D$ and falls to half at $x=1.5 D$ with only fourth of it at $x=2 D$. A peak value of TI can be observed at the top blade tip height. However, the prospective peak value at the down tip height is missing due to wake interaction of the rotor and the pile's wakes.

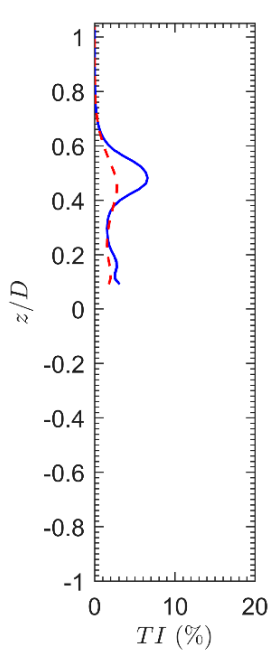

(a)



(b)

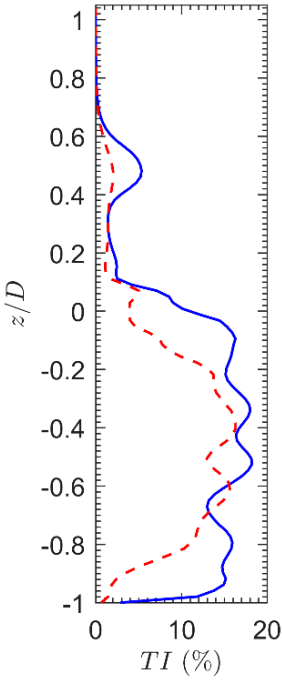

(c)

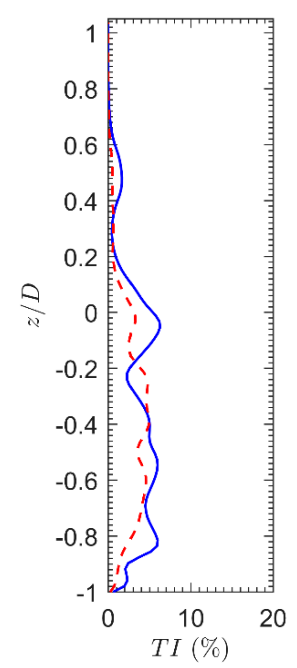

(d)

Figure 17. TI calculated using both method in the vertical direction from $x=0.5 D$ to $2 D$. (a) $x=0.5 D$. (b) $x=1 D$. (c) $x=1.5 D$. (d) $x=2 D$. Dashed line: the AL method. Solid line: the FRG method. 


\subsection{Vorticity}

Normalized vorticity $(\Omega / 2 \omega, \Omega$ is vorticity and $\omega$ is rotation speed of turbine rotor) calculated from both the AL method and FRG method in the horizontal plane across the hub center is shown in Figure 18. From the results of both methods, it can be seen that distinct tip vortices are shed with a longitudinal spacing which persist for some distance downstream. These vortices then merge into a vortex sheet which dissipates further downstream. This trend is well captured by both numerical methods. However, the FRG method obtains a higher level in the vorticity magnitude, as shown in Figure 18b. This is due to the fact that the geometry of the rotor was not fully taken into consideration in the AL method. As a result, vortices caused by differences between suction side and pressure side of a blade could not be generated in the AL method.

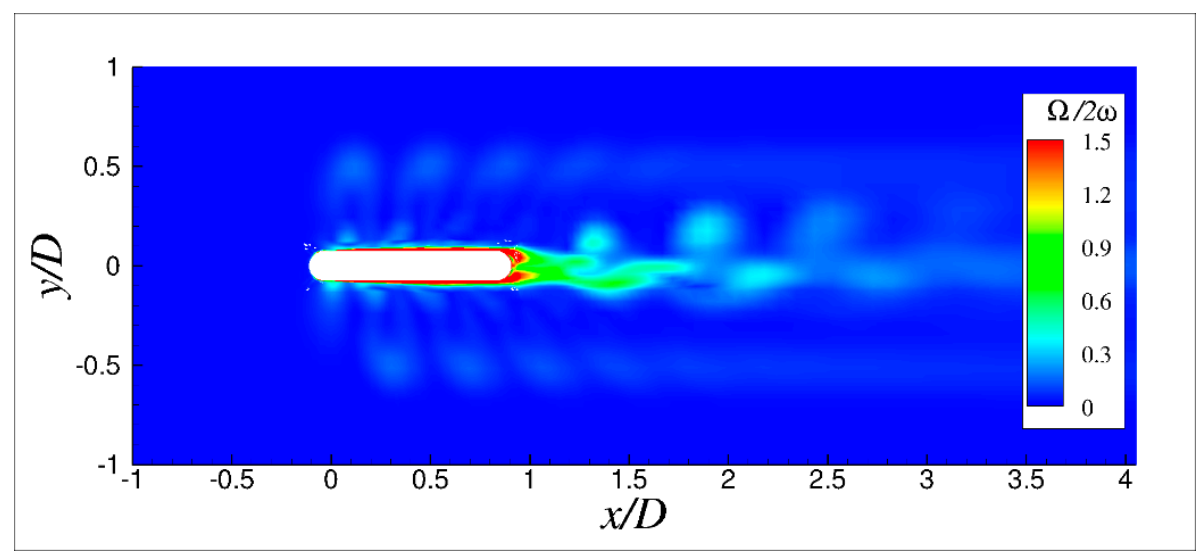

(a)

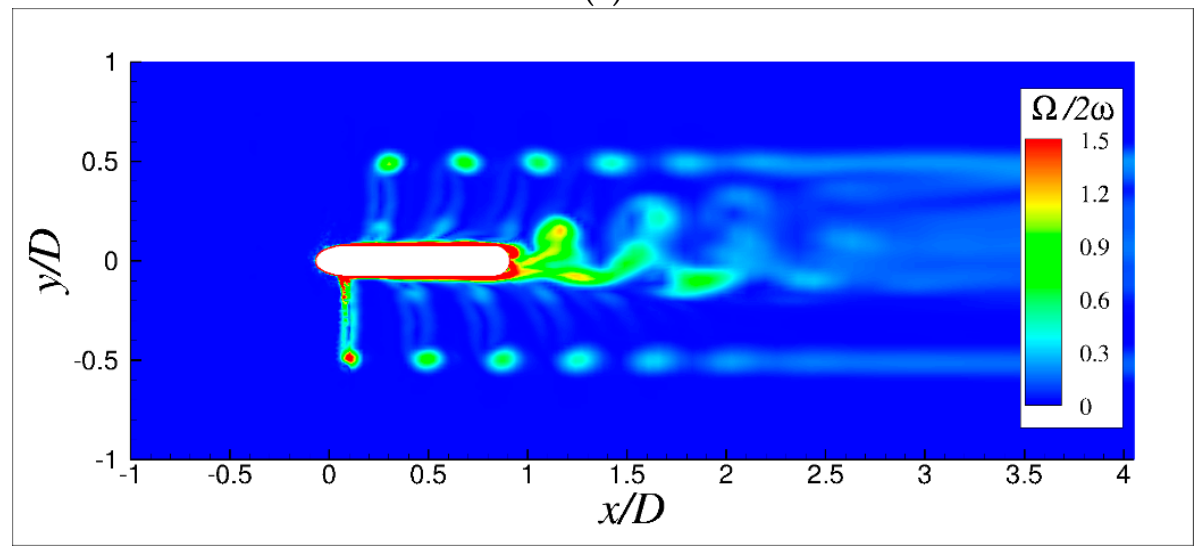

(b)

Figure 18. Vorticity distribution in the horizontal plane across the hub center. (a) The AL method. (b) The FRG method.

Figure 19 presents normalized vorticity distribution in the vertical plane across the hub center from the AL method and the FRG method. A high vorticity level can be observed behind the pile due to the shedding vortices from the monopile. The range of this high vorticity is within $x=2 D$. It is also found that the FRG method still gains a bit higher value here, compared with the AL method. Figure 20 shows the iso-surface of vorticity magnitude. It shows a strong interaction between the blade tip vortices and vortices shed from the pile. This strong interaction results in the asymmetry between 3- $D$ vorticity distribution above and beneath the nacelle. 


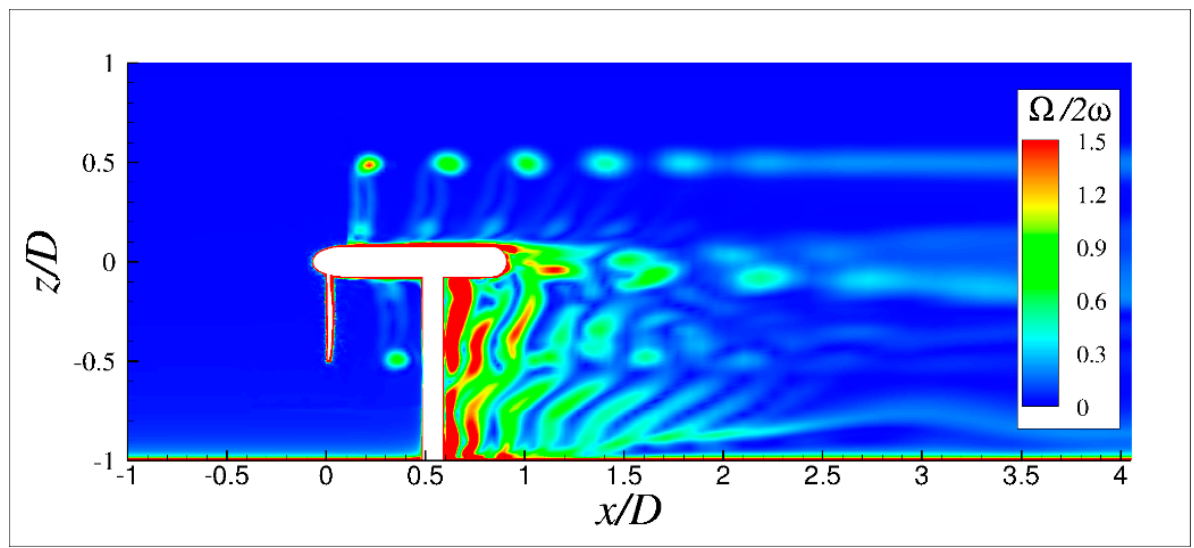

(a)

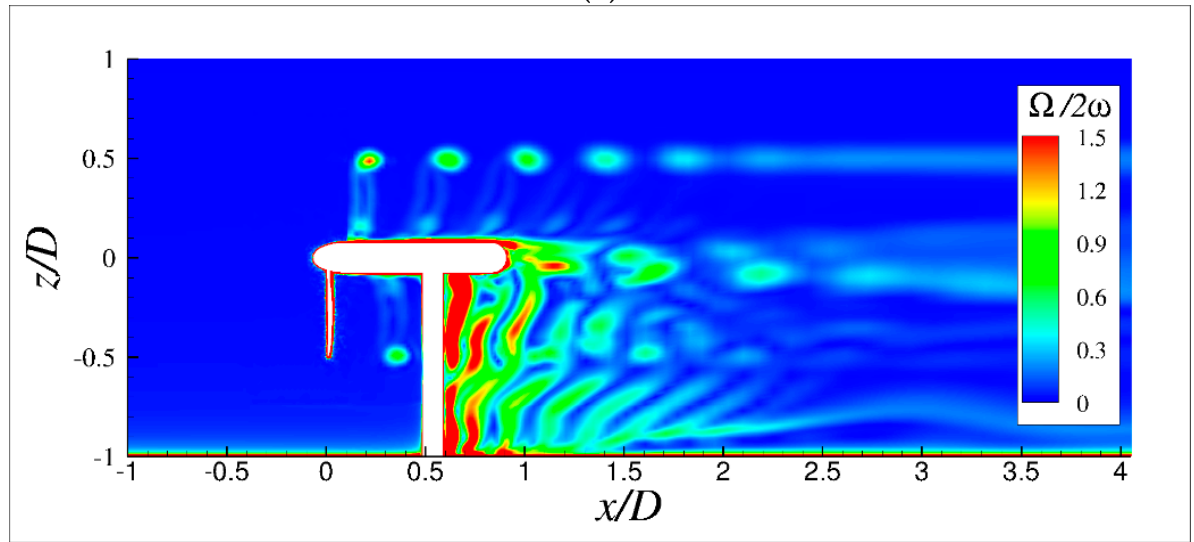

(b)

Figure 19. Vorticity distribution in the vertical plane across the hub center. (a) The AL method. (b) The FRG method.

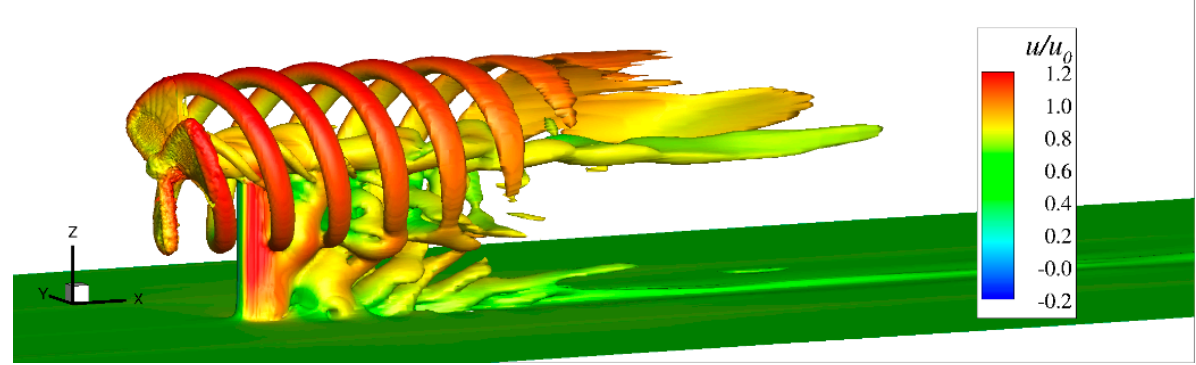

Figure 20. Iso-surface of vorticity from the FRG method with color showing the velocity magnitude.

\section{Discussion}

This study carries out a numerical simulation of a pile-supported TST using both the AL method and the FRG method based on the open-source CFD library OpenFOAM. First, the details in physical experimental set-up and numerical simulations are described. Then, a mesh refinement study is conducted to guarantee the accuracy of numerical results. Results in terms of velocity distribution, TI level and vorticity obtained by the AL method and FRG method are presented and compared.

The trend in velocity distribution in the longitudinal and lateral directions is well captured by both the methods. The resulting Gaussian distribution of velocity magnitude in the far wake is consistent with previous studies [21,40]. The AL method gives a better agreement with experimental data generally. The FRG method results in an asymmetrical stream-wise velocity distribution in the lateral direction, while the AL method obtains a symmetrical one. In terms of circumferential velocity, 
the AL method obtains a lower magnitude, compared with the FRG method. Underestimation of circumferential velocity in the AL method is also confirmed in [33]. These differences in velocity distribution between the AL method and FRG method are mainly due to the nature of the AL method [33]. As in the AL method, the rotor geometry is not included during the simulation. Water flows through the space directly where should be occupied by blades.

Comparisons of TI distribution in the lateral and vertical direction within $x=2 D$ are discussed. The FRG method obtains a higher TI level in this region, similar to [30]. In the AL method, the blade is replaced with a line and the blade boundary layer is not resolved. Therefore, the turbulence generated in the boundary layer and fed to the flow in the real case is ignored [33]. Besides, the AL method also fails to simulate the peak values of TI appearing in the downstream of the blade tips, which means the tip effects are not fully considered due to the missing rotor geometry in the AL method.

Both the AL method and FRG method reproduce the distribution of vortices in the downstream well. Vortices shedding from blade tips persist in a certain distance and then merge into a vorticity sheet in the downstream. This is consistent with previous research [34]. However, the underestimation of vorticity using the AL method can also be seen, since there is no explicit suction side and pressure side of a blade as that in the FRG method.

The presence of a monopile foundation has a significant impact on the flow field within $x=2 D$. The pile foundation introduces a region right behind it with low velocity, high TI and vorticity level. These features are similar to the wake behind a single pile [41]. However, flow field in this region results from interaction of wakes of the monopile and the rotating rotor. The combination of these wakes is of significance [32,42] in consideration of seabed scour around the monopile.

Overall, the AL method and the FRG method obtain similar patterns of flow features in the wake of a TST, although the AL method tends to underestimate the circumferential velocity, TI and vorticity slightly. This underestimation is due to the missing rotor geometry in the AL method. Nevertheless, the AL method is still an effective method in simulating the wake of a TST. Besides, further improvements, such as using an advanced turbulence model [26], could be conducted to minimize this effect. Another future research is to study wake interaction between a turbine rotor and its foundation, especially their influence on the seabed.

\section{Conclusions}

We draw the following conclusions from the above numerical simulations of the pile-supported TST:

- Both the AL method and the FRG method are capable of predicting mean stream-wise velocity distribution in the downstream filed with acceptable accuracy. Compared with the FRG method, the AL method generally gives a better agreement with experimental data, especially in the range of $x=2 \mathrm{D}$ to $6 \mathrm{D}$.

- The AL method and the FRG method give similar predictions of flow features, including the circumferential velocity, TI level and vorticity, in the downstream field, although the AL method tends to underestimate them slightly.

- Considering that the AL method saves much more computing time and can also obtain key flow features in the wake, the AL method can be regarded as a useful and effective method in reproducing the wake of a TST.

- The existence of the supporting structure has a significant influence on wake properties of the TST, especially in the near wake region within 2D downstream. It results in a region behind it with a low velocity, and high TI and vorticity level.

Author Contributions: Conceptualization, J.-s.Z.; Formal analysis, X.-f.L.; Funding acquisition, J.-s.Z.; Investigation, J.Z.; Methodology, Y.-q.Z.; Resources, J.Z.; Software, Y.-q.Z.; Supervision, J.-s.Z.; Validation, X.-f.L. and S.L.; Writing—original draft, X.-f.L. and S.L.; Writing—review and editing, J.-s.Z.

Funding: This research was funded by the National Natural Science Foundation of China (NO. 51809083), Natural Science Foundation of Jiangsu Province (NO. BK20180504), the Fundamental Research Funds for 
the Central Universities of China (No. 2017B696X14), the marine renewable energy research project of State Oceanic Administration (No. GHME2015GC01), the Postgraduate Research and Practice Innovation Program of Jiangsu Province, China (No. KYCX17_0048) and the Fundamental Research Funds for the Central Universities (No. 2017B696X14).

Acknowledgments: The authors are grateful for support from Da-wei Guan and College of Civil Engineering in Shandong Jiaotong University.

Conflicts of Interest: The authors declare no conflicts of interest.

\section{Nomenclature}

\begin{tabular}{|c|c|}
\hline TST & tidal stream turbine \\
\hline TI & turbulence intensity \\
\hline CFD & computational fluid dynamics \\
\hline $\mathrm{AD}$ & actuator discs \\
\hline BEM & blade element momentum \\
\hline FRG & full rotor geometry \\
\hline AL & actuator line \\
\hline LES & large eddy simulation \\
\hline HPC & High Performance Computing \\
\hline RAM & random access memory \\
\hline RANS & Reynolds-averaged Navier-Stokes \\
\hline SST & shear stress transport \\
\hline ADV & acoustic Doppler velocimeter \\
\hline SNR & signal to noise ratio \\
\hline PISO & Pressure-Implicit with Splitting of Operators \\
\hline CFL & Courant-Friedrichs-Lewy \\
\hline NACA & the National Advisory Committee for Aeronautics \\
\hline $\mathrm{D}$ & rotor diameter \\
\hline $\mathrm{R}$ & rotor radius \\
\hline $\mathrm{r}$ & radial location of local blade cross-sections \\
\hline c & chord length of local blade cross-sections \\
\hline h & water depth \\
\hline TSR & tip speed ratio \\
\hline$f_{i}$ & source term in the i direction due to the blades' forces \\
\hline $\mathrm{d} F_{Z}$ & axial forces imposed on a blade element \\
\hline $\mathrm{d} F_{\theta}$ & rotational forces imposed on a blade element \\
\hline$\rho$ & water density \\
\hline$d r$ & width of a blade element \\
\hline$V_{\text {vel }}$ & relative velocity \\
\hline$c_{l}$ & lift coefficient \\
\hline$c_{d}$ & drag coefficient \\
\hline$R_{e}$ & sectional Reynolds number \\
\hline$\alpha$ & local angle of attack \\
\hline$\omega$ & blade's rotation speed \\
\hline$\gamma$ & blade element's twist angle \\
\hline$\phi$ & flow angle \\
\hline$V_{Z}$ & flow velocity in the $\mathrm{Z}$ direction \\
\hline$V_{\theta}$ & flow velocity in the $Z$ and $\theta$ direction \\
\hline$\eta$ & projection width \\
\hline$\epsilon$ & parameters \\
\hline$\vec{r}$ & projection distance \\
\hline$u$ & local stream-wise flow velocity \\
\hline$U$ & mean approach velocity \\
\hline$F_{r}$ & Froude number \\
\hline$g$ & acceleration due to gravity \\
\hline AMI & arbitrary mesh interface \\
\hline $\mathrm{y}^{+}$ & non-dimensional wall distance \\
\hline$x$ & longitudinal distance away from the rotor center \\
\hline$y$ & transverse distance away from the rotor center \\
\hline$z$ & vertical distance away from the rotor center \\
\hline RMSE & root mean square error \\
\hline$\Omega$ & vorticity \\
\hline
\end{tabular}




\section{References}

1. Mejia-Olivares, C.J.; Haigh, I.D.; Wells, N.C.; Coles, D.S.; Lewis, M.J.; Neill, S.P. Tidal-stream energy resource characterisation for the Gulf of California, México. Energy 2018, 156, 481-491. [CrossRef]

2. Bonar, P.A.J.; Schnabl, A.M.; Lee, W.K.; Adcock, T.A.A. Assessment of the Malaysian tidal stream energy resource using an upper bound approach. J. Ocean Eng. Mar. Energy 2018, 4, 99-109. [CrossRef]

3. Lamy, J.V.; Azevedo, I.L. Do tidal stream energy projects offer more value than offshore wind farms? A case study in the United Kingdom. Energy Policy 2018, 113, 28-40. [CrossRef]

4. Lin, J.; Lin, B.L.; Sun, J.; Chen, Y.L. Modelling hydrodynamic processes in tidal stream energy extraction. J. Hydrodyn. 2016, 28, 1058-1064. [CrossRef]

5. Zheng, J.; Dai, P.; Zhang, J. Tidal stream energy in China. Procedia Eng. 2015, 116, 880-887. [CrossRef]

6. Payne, G.S.; Stallard, T.; Martinez, R. Design and manufacture of a bed supported tidal turbine model for blade and shaft load measurement in turbulent flow and waves. Renew. Energy 2017, 107, 312-326. [CrossRef]

7. Martinez, R.; Payne, G.S.; Bruce, T. The effects of oblique waves and currents on the loadings and performance of tidal turbines. Ocean Eng. 2018, 164, 55-64. [CrossRef]

8. Lewis, M.; Neill, S.P.; Robins, P.E.; Hashemi, M.R. Resource assessment for future generations of tidal-stream energy arrays. Energy 2015, 83, 403-415. [CrossRef]

9. González-Gorbeña, E.; Qassim, R.Y.; Rosman, P.C.C. Optimisation of hydrokinetic turbine array layouts via surrogate modelling. Renew. Energy 2016, 93, 45-57. [CrossRef]

10. Funke, S.W.; Kramer, S.C.; Piggott, M.D. Design optimisation and resource assessment for tidal-stream renewable energy farms using a new continuous turbine approach. Renew. Energy 2016, 99, 1046-1061. [CrossRef]

11. Funke, S.W.; Farrell, P.E.; Piggott, M.D. Tidal turbine array optimisation using the adjoint approach. Renew. Energy 2014, 63, 658-673. [CrossRef]

12. Stansby, P.; Stallard, T. Fast optimisation of tidal stream turbine positions for power generation in small arrays with low blockage based on superposition of self-similar far-wake velocity deficit profiles. Renew. Energy 2016, 92, 366-375. [CrossRef]

13. McCombie, P.; Sullivan, P. Optimisation of tidal power arrays using a genetic algorithm. Proc. ICE-Energy 2013, 166, 19-28.

14. Funke, S.W.; Thyng, K.M.; Roc, T. Standard methodology for tidal array project optimisation: An idealized study of the Minas Passage. In Proceedings of the 11th European Wave and Tidal Energy Conference-EWTEC, Nantes, France, 6-11 September 2015.

15. Myers, L.E.; Bahaj, A.S. An experimental investigation simulating flow effects in first generation marine current energy converter arrays. Renew. Energy 2012, 37, 28-36. [CrossRef]

16. Ahmadian, R.; Falconer, R.A. Assessment of array shape of tidal stream turbines on hydro-environmental impacts and power output. Renew. Energy 2012, 44, 318-327. [CrossRef]

17. Nash, S.; Phoenix, A. A review of the current understanding of the hydro-environmental impacts of energy removal by tidal turbines. Renew. Sustain. Energy Rev. 2017, 80, 648-662. [CrossRef]

18. Mycek, P.; Gaurier, B.; Germain, G.; Pinon, G.; Rivoalen, E. Experimental study of the turbulence intensity effects on marine current turbines behaviour. Part I: One single turbine. Renew. Energy 2014, 66, 729-746. [CrossRef]

19. Mycek, P.; Gaurier, B.; Germain, G.; Pinon, G.; Rivoalen, E. Experimental study of the turbulence intensity effects on marine current turbines behaviour. Part II: Two interacting turbines. Renew. Energy 2014, 68, 876-892. [CrossRef]

20. Chamorro, L.P.; Hill, C.; Morton, S.; Ellis, C.; Arndt, R.E.A.; Sotiropoulos, F. On the interaction between a turbulent open channel flow and an axial-flow turbine. J. Fluid Mech. 2013, 716, 658-670. [CrossRef]

21. Tedds, S.C.; Owen, I.; Poole, R.J. Near-wake characteristics of a model horizontal axis tidal stream turbine. Renew. Energy 2014, 63, 222-235. [CrossRef]

22. Stallard, T.; Feng, T.; Stansby, P.K. Experimental study of the mean wake of a tidal stream rotor in a shallow turbulent flow. J. Fluids Struct. 2015, 54, 235-246. [CrossRef]

23. Chen, Y.; Lin, B.; Lin, J.; Wang, S. Experimental study of wake structure behind a horizontal axis tidal stream turbine. Appl. Energy 2017, 196, 82-96. [CrossRef]

24. Harrison, M.E.; Batten, W.M.J.; Myers, L.E.; Bahaj, A.S. A comparison between CFD simulations and experiments for predicting the far wake of horizontal axis tidal turbines. IET Renew. Power Gener. 2010, 4, 613. [CrossRef] 
25. Thiébot, J.; Guillou, S.; Nguyen, V.T. Modelling the effect of large arrays of tidal turbines with depth-averaged Actuator Disks. Ocean Eng. 2016, 126, 265-275. [CrossRef]

26. Shives, M.; Crawford, C. Adapted two-equation turbulence closures for actuator disk RANS simulations of wind \& tidal turbine wakes. Renew. Energy 2016, 92, 273-292.

27. Batten, W.M.; Harrison, M.E.; Bahaj, A.S. Accuracy of the actuator disc-RANS approach for predicting the performance and wake of tidal turbines. Philos. Trans. 2013, 371, 20120293. [CrossRef] [PubMed]

28. Edmunds, M.; Williams, A.J.; Masters, I.; Croft, T.N. An enhanced disk averaged CFD model for the simulation of horizontal axis tidal turbines. Renew. Energy 2017, 101, 67-81. [CrossRef]

29. Guo, Q.; Zhou, L.; Wang, Z. Comparison of BEM-CFD and full rotor geometry simulations for the performance and flow field of a marine current turbine. Renew. Energy 2015, 75, 640-648. [CrossRef]

30. Liu, J.; Lin, H.; Purimitla, S.R. Wake field studies of tidal current turbines with different numerical methods. Ocean Eng. 2016, 117, 383-397. [CrossRef]

31. Nuernberg, M.; Tao, L. Three dimensional tidal turbine array simulations using OpenFOAM with dynamic mesh. Ocean Eng. 2018, 147, 629-646. [CrossRef]

32. Chen, L.; Lam, W.H. Slipstream between marine current turbine and seabed. Energy 2014, 68, 801-810. [CrossRef]

33. Baba-Ahmadi, M.H.; Dong, P. Numerical simulations of wake characteristics of a horizontal axis tidal stream turbine using actuator line model. Renew. Energy 2017, 113, 669-678. [CrossRef]

34. Baratchi, F.; Jeans, T.L.; Gerber, A.G. Actuator line simulation of a tidal turbine in straight and yawed flows. Int. J. Mar. Energy 2017, 19, 235-255. [CrossRef]

35. Bachant, P.; Goude, A.; Wosnik, M. Actuator line modeling of vertical-axis turbines. arXiv, 2016; arXiv:1605.01449.

36. Weller, H.G.; Tabor, G.; Jasak, H.; Fureby, C. A tensorial approach to computational continuum mechanics using object-oriented techniques. Comput. Phys. 1998, 12, 620-631. [CrossRef]

37. The OpenFOAM Foundation OpenFOAM v6 User Guide. Available online: https://cfd.direct/openfoam/ user-guide (accessed on 5 November 2018).

38. Goring, D.G.; Nikora, V.I. Despiking acoustic Doppler velocimeter data. J. Hydraul. Eng. 2002, 128, 117-126. [CrossRef]

39. Mcnaughton, J.; Afgan, I.; Apsley, D.D.; Rolfo, S.; Stallard, T.; Stansby, P.K. A simple sliding-mesh interface procedure and its application to the CFD simulation of a tidal-stream turbine. Int. J. Numer. Methods Fluids 2014, 74, 250-269. [CrossRef]

40. Lam, W.H.; Chen, L.; Hashim, R. Analytical wake model of tidal current turbine. Energy 2015, 79, 512-521. [CrossRef]

41. Rogan, C.; Miles, J.; Simmonds, D.; Iglesias, G. The turbulent wake of a monopile foundation. Renew. Energy 2016, 93, 180-187. [CrossRef]

42. Tatum, S.C.; Frost, C.H.; Allmark, M.; O’Doherty, D.M.; Mason-Jones, A.; Prickett, P.W.; Grosvenor, R.I.; Byrne, C.B.; O'Doherty, T. Wave-Current interaction effects on tidal stream turbine performance and loading characteristics. Int. J. Mar. Energy 2016, 14, 161-179. [CrossRef]

(C) 2019 by the authors. Licensee MDPI, Basel, Switzerland. This article is an open access article distributed under the terms and conditions of the Creative Commons Attribution (CC BY) license (http:// creativecommons.org/licenses/by/4.0/). 